Article

\title{
Evolution of the Size Distribution of an Emulsion under a Simple Shear Flow
}

\author{
Jairo M. Leiva * and Enrique Geffroy \\ Instituto de Investigaciones en Materiales, Universidad Nacional Autónoma de México, Ciudad Universitaria, \\ 04510 Cd. de México, CDMX, Mexico; geffroy@unam.mx \\ * Correspondence: eleiva@iim.unam.mx; Tel.: +52-55-5622-4632
}

Received: 25 April 2018; Accepted: 20 June 2018; Published: 25 June 2018

\begin{abstract}
Understanding the rheology of immiscible liquids mixtures, as well as the role played by its micro-structures are important criteria for the production of new materials and processes in industry. Here, we study changes over time of the droplet size distributions of emulsions induced by slow shearing flows. We observe that the initial heterogeneous microstructure may evolve toward more complex structures (such as bimodal distribution) as a result of coalescence and rupture of droplets. These dynamic structures were produced using a flow cell made up of two parallel disks, separated by a gap of $100 \mu \mathrm{m}$. The steady rotation of the lower disk generates a simple shear flow of $\dot{\gamma}=0.75 \mathrm{~s}^{-1}$, during $\sim 400 \mathrm{~s}$. After a brief rest time, this procedure was repeated by applying a step ramp until the maximum shear rate of $4.5 \mathrm{~s}^{-1}$ was reached, using step increments of $0.75 \mathrm{~s}^{-1}$. During the last portion of the flow and during the rest time in between flows, structures of emulsions were characterized. Initially, a broad single-peak distribution of drops was observed, which evolved toward a rather narrower bimodal distribution, at first due to the coalescence of the smaller droplets and subsequently of the larger drops. The rupture of drops at higher shear rates was also observed. The observed evolutions also presented global structures such as "pearl necklaces" or "bands of particles", the latter characterized by alternating bands of a high density of particles and regions of the continuous phase with only a few droplets. These changes may indicate complex, time-dependent rheological properties of these mixtures.
\end{abstract}

Keywords: emulsion microstructure; drop size distribution; monomodal-bimodal distributions

\section{Introduction}

Emulsions are of great relevance for a variety of applications in food, pharmaceuticals, adhesives, cosmetics, plastics, fertilizers, and petroleum recovery industries, inasmuch as mixtures of fluids provide a wider range of properties in their final products. However, the properties of the mixtures depend, to a great extent, on the microstructure of the emulsion, which is, in turn, the result of the history of the flow. Therefore, most rheological properties must be considered as dynamical properties, which may depend on the structure of the emulsion in a nonlinear manner.

For emulsions, the time evolution of their rheological properties can depend on a number of factors, such as fluid properties-i.e., the fluid viscosities and the interfacial energy-the particle size and shape, volume fractions of the phases, and other subtler effects, such as the anisotropic distribution of particles that the imposed flow can induce [1]. In the literature, we find studies focused on the effects characterized by important processes, such as the role of coalescence-rupture [2-4] of drops or parameters (e.g., the viscosity ratio [5,6], the shear rate [7-9], and the volume fraction $[10,11]$ ). Nevertheless, much remains to be explored mainly because a complete understanding of the observed phenomena [12] is still lacking. This is especially relevant when the structure evolution shows multiple well-defined structures as time passes under a flow field, particularly, when global anisotropic structural 
changes are induced at higher shear rates. Despite the possible relevance of the latter, here, we address the changes in the microstructure of the emulsion at low shear rates and in the relevance to the rheology of the emulsion. This behavior corresponds to the low, dimensionless time evolutions induced by slow shearing flows presented in this work.

\subsection{Theoretical Background}

There are many results published on the effects of the history of the shear rate (e.g., on the deformation, breakup of a single drop, or coalescence of quasi-equal size drops). The pioneering work of G. I. Taylor [13] about the slight deformation of a single drop establishes experimentally that two dimensionless numbers mainly determine the drop deformation under a linear flow: the capillary number and the viscosity ratio. Larger capillary numbers induce larger drop deformations, and viscous drops require a higher shear rate or capillary number in order to deform significantly.

The capillary number is given by Equation (1)

$$
C a=\frac{\eta_{m} R \dot{\gamma}}{\sigma}
$$

where $\eta_{m}, R, \dot{\gamma}$, and $\sigma$ represent the viscosity of the fluid matrix, the radius of the drop, the applied shear rate, and the interfacial tension coefficient between phases, respectively.

The viscosity ratio between the two phases is given by Equation (2)

$$
p=\frac{\eta_{d}}{\eta_{m}}
$$

where $\eta_{d}$ is the viscosity of the disperse fluid, and $\eta_{m}$ is the viscosity of the continuum fluid or matrix.

When studying emulsions under flow (besides deformation of drops), other phenomena can frequently be observed, such as coalescence of drops [4], break up of drops [6], or capture of a rather small drop by another significantly larger drop [14]. When applying a larger shear rate to the emulsion, these phenomena may be observed and are frequently the main source of the observed dynamical changes of the size distributions of drops; although, each phenomenon depends most likely on different physical mechanisms. Under weak flows, coalescence of small, equal-sized drops is observed, and the rupture of drops occurs at higher rates of deformation, especially with low viscosity fluids. Research reporting large induced deformations under shearing flows, even beyond a critical drop size (up to break-up into two or multiple droplets), are given in [15-17]. The growth of drops through the capture of much smaller, nearby droplets-by a mechanism that appears to resemble an Oswald ripening process — can be readily produced but requires a rather broad size distribution, including large drops. Finally, spatially varying distributions of particles induced by flow have been observed as well, but its relation to the former phenomena is less well documented. Here, we attempt to describe a robust technique to evaluate the slow-shear-flow phenomena, which generally modifies the observed rheology of an emulsion in a rather complex and nonlinear manner.

\subsubsection{Coalescence in Slow Flows}

Here, the coalescence mechanism occurs mainly in the weakest of flows. During this process, two drops may coalesce if they spend enough time in close proximity. Thus, a dimensionless time, $\tau$, indicative of the minimum time required for a high probability of coalescence (or its efficiency) can be calculated as

$$
\tau=\dot{\gamma} t_{e}
$$

where $t_{e}$ corresponds to the duration of imposed flow of the experiment, while the shear rate, $\dot{\gamma}$, is proportional to the rate of collisions of drops of a given size. Please note that it is customary to consider Equation (3) as a deformation measure, but here, we prefer to associate the inverse of this dimensionless number to an efficacy or efficiency of coalescence. Dimensionless deformation measures 
are most appropriate when studying fluids with a homogeneous and continuous micro-structure, such as polymer solutions (with an associated characteristic time-scale spectrum), etc. In contrast, for our experiments, a dimensionless time is more closely associated with inverse frequency of events. This may provide a better understanding between the statistics of the drops distributions and the experimental conditions. The inverse time for shear rate is associated here with a value proportional to the frequency of collision of drops and is not relevant as a measure of rate of deformation.

This interpretation allows us to compare different flow regimes, assuming that other present phenomena remain stationary. The proportionality constant for the rate of collisions is a rather complex function of the hydrodynamics of multiple interacting drops. For isolated pairs of drops (i.e., very dilute emulsions), the film drainage model is frequently used, which is more suited for non-deformable surfaces (i.e., drops of high viscosity) $[18,19]$.

For emulsions, mean field values for the drainage model are difficult to calculate. In contrast, when evaluating the evolution of the size distributions under flow, coalescence between the smallest of drops can be inferred rather easily, because the rate of decrease of frequency for the smallest drops is about twice the rate of increase of frequency of drops with double their volume. The same is true for the larger-size drops of multimodal distribution, as will be shown subsequently. Thus, the coalescence rate can be established by the product of the frequency of the drops' collision times the efficiency of coalescence [20].

For emulsions with a high fraction of the disperse phase-characterized by a broad size distribution and under weak shearing flows-the experimental information is difficult to interpret, due mainly to a high density of very small drops, especially at the onset of the flow. It is also evident that an upper limit to the drop size (spheroidal drops and for a given shear rate) exists when the kinetics of the rupture of the drops competes with the coalescence phenomenon, and the changes of the distribution of small drops vanish almost completely [21]. Given that the capillary number increases for the larger drops, then a maximum size exists, where coalescence dominates and rupture kinetics begin, cancelling each other out. In the work of Grizzuti and Minale, it is suggested that the two processes coexist in the same system [19-27]. Therefore, a second critical capillary number should be observed-associated with the transition of coalescence to rupture-and defined when drops increasing in size undergo a breakage process $[11,12,23,24]$. The purpose of this work is to clarify the presence of coalescence and rupture processes under shearing flows by studying the evolution of the drop size distribution of the dispersed phase.

\subsubsection{Breakup of Droplets under a Shearing Flow}

The so-called critical capillary number required for the rupture of a vesicle, $C a_{r u p}$, appears to depend principally on $p$ for simple shear flows, as shown by Grace [16] and De Bruijn [17]. A broad set of data for drops deformation and break up, including a large class of two-dimensional (2D)-flows-covering from simple shear up to a purely elongational flow-was provided by Bentley and Leal [28]. More recently, for emulsions subjected to simple shear flows, Jansen [29] has shown that the critical capillary number decreases with increments of the fraction of the disperse phase: $C a_{\text {rup }}(p, \phi)$. Droplet-breaking mechanisms and shapes of Newtonian liquid droplets have been extensively studied. If $\mathrm{Ca}<<1$, the drop shape is slightly ellipsoidal, depending on $p$, and aligned at an orientation angle of $45^{\circ}$ with respect to the direction of flow. As the capillary number increases, the steady state elongation grows, and the drop rotates aligning itself along the direction of flow. For higher capillary numbers, beyond the critical value, rupture is observed with the breaking mode depending on the viscosity ratio. For $p<1$, the drops assume an elongated highly cusped form, from which small drops (the so-called tip streaming phenomenon) are launched. For $p$ approximately equal to 1 , the central portion of the droplet forms a neck (or necks) followed by the breaking up into two daughter-droplets, with small satellite droplets between them. $\mathrm{Ca}>\mathrm{C} a_{\text {rup }}$, droplets are deformed into long, thin filaments that eventually break up through the instability of the capillary wave mechanism. These mechanisms become more complex as the density of disperse phase drops increases. 


\section{Materials and Methods}

\subsection{Constituents and Preparation of Emulsions}

Two immiscible fluids were prepared as the emulsion disperse-continuum components, looking for a pair of liquids with high viscosities and equal densities: an aqueous solution, as the dispersed phase, and a mixture of alkanes. The aqueous solutions is (w/W) $10 \mu \mathrm{M}$ polyethylene oxide (with a viscosity-averaged molecular weight of $M_{v} \sim 1,000,000$, Sigma-Aldrich CAS\#372781, Sigma-Aldrich, St. Louis, MO, USA) in $97 \%$ ultra-pure water (resistivity $\geq 18.2 \mathrm{M} \Omega \cdot \mathrm{cm} ; \rho=0.997 \mathrm{~g} / \mathrm{mL}$ )) and 3\% 2-propanol (Sigma-Aldrich CAS\#190764, $\geq 99.5 \%$ Reagent grade). The continuum phase is a mixture of eicosane (Sigma-Aldrich CAS\#219274), heptadecane (Sigma-Aldrich CAS\#128503), 1,2,4-trichlorobenzene (Sigma-Aldrich CAS\#132047), and polybutadiene (Sigma-Aldrich CAS\#181382). The alkane fluid is prepared by first mixing $7.56 \%$ eicosane with $39.69 \%$ heptadecane in a glass bottle, while maintaining it at $30{ }^{\circ} \mathrm{C}$, and then adding 46.5\% 1,2,4-trichlorobenzene and $6.25 \%$ polybutadiene $\left(M_{n} \sim 200,000\right)$. The viscosities of the fluids were measured with an ARES G2 Rheometer (TA Instruments, New Castle, DE, USA) using the concentric cylinder geometry. The aqueous phase has a viscosity of $0.57 \mathrm{~Pa} \cdot \mathrm{s}$ and the continuum phase a viscosity of $2.08 \mathrm{~Pa} \cdot \mathrm{s}$ at $30{ }^{\circ} \mathrm{C}$; the viscosity ratio is $p=0.27$. The densities $\left(\mathrm{g} / \mathrm{cm}^{3}\right.$, at $\left.30{ }^{\circ} \mathrm{C}\right)$ are 0.98 and 0.95 for the aqueous phase and the continuum phase, respectively. By using 2-propanol (aqueous solution) and trichlorobenzene (alkane mixture), the density of the two fluids can be adjusted to minimize sedimentation in the emulsion. The interfacial tension $\sigma$ was determined by the deformed drop retraction (DDR) method, as described by Guido and Villone [30], using the optical shear cell CSS450, which is the same cell that we use in this work. The measured averaged surface tension is $0.11 \mathrm{mN} / \mathrm{m}$, evaluated for a set of 11 drops of aqueous fluid $(40 \%)$ in the oil phase.

The emulsion was prepared by mixing a $50 \mathrm{wt} \%$ oil phase with $50 \mathrm{wt} \%$ water phase using an homogenizer (Omni Inc., Kennesaw Georgia, GA, USA) with a generator probe $10 \mathrm{~mm} \times 95 \mathrm{~mm}$ fine saw tooth (SKU\#15051), spinning at $3000 \mathrm{rpm}$ during $300 \mathrm{~s}$, and at a constant temperature of $30{ }^{\circ} \mathrm{C}$. Afterwards, the emulsion was placed in glass tubes ( $5 \mathrm{~mm}$ inner diameter) at rest for $48 \mathrm{~h}$ and at $30^{\circ} \mathrm{C}$. In this way, the stability of the emulsion was visually evaluated after $48 \mathrm{~h}$, ensuring that trapped air bubbles were removed. The sample of the emulsion was placed on the bottom plate of the flow cell, and the top plate was carefully placed on top, while the plates were being slowly compressed (squeezed) to reduce the separation until reaching a gap of $100 \mu \mathrm{m}$. This compression process reduces residual stresses while maintaining the homogeneity of the sample. Following loading, the cell was allowed to relax for a period of $\sim 600 \mathrm{~s}$. All flow experiments started with an initial pre-conditioning of the emulsion to eliminate possible residual stresses by subjecting it to a shear rate of $0.075 \mathrm{~s}^{-1}$ for $\sim 500 \mathrm{~s}$. During this initial flow, the drop size data was dominated by a large count of very small drops-with diameters below the resolution of the optical arrangement $\leq 5 \mu \mathrm{m}$-and no comparison with the drop distribution after the loading of the cell is considered reliable. At this instant, we set $\tau \equiv 0$.

Subsequently, a ramp sequence of constant steady shear flows was applied for each sample. Each step of the ramp consisted of a steady flow applied during $\sim 400 \mathrm{~s}$, followed by a no-flow rest time of $\sim 18 \mathrm{~s}$, sufficiently long for drops to attain a spherical shape. The initial step of the ramp began with a flow of $\dot{\gamma}=0.75 \mathrm{~s}^{-1}$, and subsequent flows stepped up by increments of $0.75 \mathrm{~s}^{-1}$ up to $4.5 \mathrm{~s}^{-1}$. Toward the end of each steady shear stress-just before the flow was stopped-and a short time after the no flow condition prevailed, a set of images was taken for the statistical analysis. For all the experiments, the no-flow rest time appears to have a negligible effect on the drop size distributions while facilitating the determination of the size of particles of a quasi-spherical shape. Thus, this multistep history of flow stresses is responsible for the changes in the distribution of drops in the sample. The influence of stopping the flow upon the global dimensionless time $\left(\tau=\dot{\gamma} t_{e}\right)$, as well as the structure of the distribution, can be considered negligible. Here, no inertia effects on the deformation of drops is expected given that the nominal Reynolds number-based on tangential velocity, cell gap, and viscosity of the continuum phase-are within $3 \times 10^{-6}-2 \times 10^{-5}$, for all shear rates. 


\subsection{Smooth Kernel Distribution Estimation}

The smoothing of all drop size frequency histograms was done with the kernel density technique in order to obtain the probability density function with a known collection of frequency points. Here, the histogram area under the curve is assumed to be 1 , and the probability of a drop diameter $d_{i}$ corresponds to the area under the curve between those two points $\left(d_{i}, d_{i}+\Delta d\right)$, where $\Delta d$ is the difference between diameters [31]. This tool provides a quick evaluation of the distribution as a continuous function; the smoothing parameter (bandwidth) used in all histograms is 1.25 [32]. We also evaluated the quality of polydispersity for the drop distributions via a polydispersity index based on the average drop size (diameter) $D_{1,0}$, the average volume size $D_{4,3}$, and the contributions of the tails of the distribution, respectively:

$$
\begin{gathered}
d_{N}=D_{1,0}=\frac{\sum_{i=1}^{\infty} n_{i} \cdot d_{i}}{\sum_{i=1}^{\infty} n_{i}}, \\
d_{V}=D_{4,3}=\frac{\sum_{i=1}^{\infty} n_{i} \cdot d_{i}^{4}}{\sum_{i=1}^{\infty} n_{i} \cdot d_{i}^{3}{ }^{\prime}} \\
\text { skewness }=\frac{n}{(n-1)(n-2)} \sum_{i=1}^{n}\left(\frac{d_{i}-\bar{d}}{s d}\right)^{3}, \\
\text { kurtosis }=\frac{n(n+1)}{(n-1)(n-2)(n-3)} \sum_{i=1}^{n}\left(\frac{d_{i}-\bar{d}}{s d}\right)^{4}-3 \frac{(n-1)^{2}}{(n-2)(n-3)} .
\end{gathered}
$$

\subsection{The Experimental Conditions}

All experiments were performed with the parallel plate geometry (Linkam CSS450, Linkam Scientific Instruments, Tadworth, UK), schematically shown in Figure 1. It consisted of two parallel quartz plates with a diameter of $36 \mathrm{~mm}$, each in contact with flat silver heaters on the outside, with an observation window located at $7.5 \mathrm{~mm}$ radial position. The motion of the lower disc imposed a shearing stress field on the emulsion. Images were captured on the vorticity-velocity plane through the $2.8 \mathrm{~mm}$ observation window. The effects of the shearing and duration of the imposed shear flow were studied using a constant rotation rate with a speed control better than $1 \%$ of the rotational speed and using a sequence of microstructure measurements at spaced events in time. In the present work, a gap spacing of $0.1 \mathrm{~mm}$ between disks and a temperature $30^{\circ} \mathrm{C}$ was used.

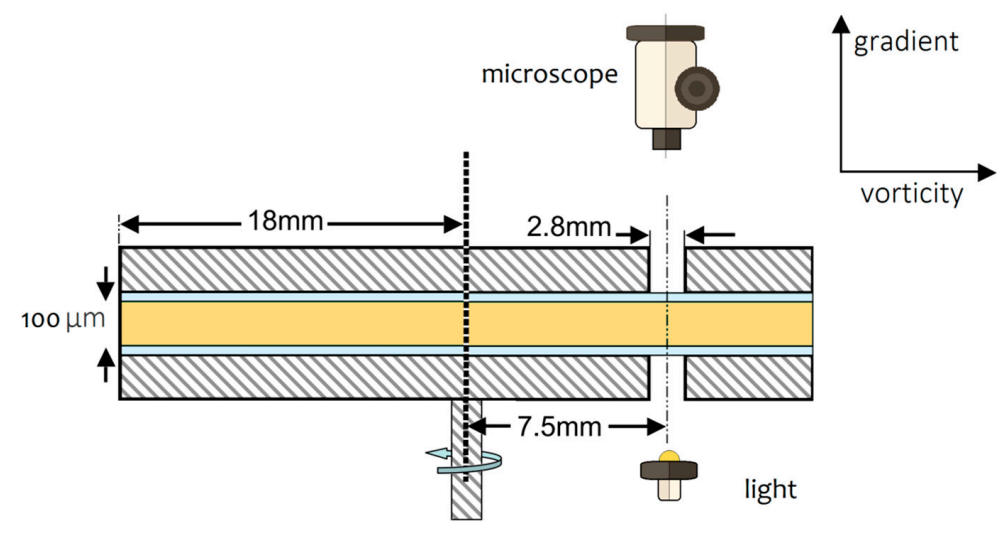

Figure 1. Schematic description of the shearing device: parallel-plate geometry with a diameter of $36 \mathrm{~mm}$ and a gap of $100 \mu \mathrm{m}$. The observation plane is described by the velocity-vorticity axes. The motion of the lower disc imposes a simple shearing stress field on the sample.

Emulsion structures were visualized using an optical microscope Nikon SMZ-U (Nikon Corp., Tokyo, Japan). Capture of images was carried out with a Nikon Digital Sight DS-2 mV camera 
in a bright field illumination arrangement. All images were processed with the Image ${ }^{\circledR}$ software (U.S. National Institutes of Health, Bethesda, MD, USA), manually and automatically. Pre-processing of drop images included assigning a threshold value, converting the image to a binary map, followed by an automatic count of drops with circularity better than $>0.94$. The statistical methods are later applied after verifying the reliability and veracity of the data obtained by the image processing. Prior to the selection of an experimental run, the image capturing process was optimized in order to reduce the emulsion turbidity and proper exposure to light [33]. Thus, a full view image with the focus plane centered on the plane of the flow field was assured. In order to visualize the evolution of the microstructure, multiple images were taken towards the end of the shearing period and after the flow stopped and fully relaxed; images were spaced at intervals of $1 \mathrm{~s}$ for statistical analysis.

In order to obtain the droplet size distributions, mainly three operations were carried out (i.e., the image acquisition, pre-processing of digital images (involving cleansing, defining drop contours, etc.), and the statistical analysis of at least two (or multiple) images) to generate the frequency histograms. The observed droplets are those present only on the focal plane normal to the direction of the velocity-gradient. The depth of field for the optical arrangement is approximately $100 \mu \mathrm{m}$, about the size of the gap between plates; thus, most drops in the flow field were observed, while the width of the field view of the microscope on the focal plane was 2-3 times larger than the captured image, thus minimizing magnification ratio variations away from the central axis.

For $\tau<0$ - that is, using a gap of $100 \mu \mathrm{m}$ during pre-shearing-images would have shown many small drops, with several of them frequently overlapping along the optical path, making the correct determination of the size or the count numbers of the drops almost impossible. The highest possible concentration (of small droplets) occurred at the onset of the flow $(\tau \equiv 0)$. At this instant, the average size of the droplets implied a tight closeness of particles, and in order to be able to distinguish every individual droplet, data was only taken after $\sim 380 \mathrm{~s}$. That is, even for the most concentrated emulsions, the image processing algorithm must assign a given size to each drop. It is only for $\dot{\gamma}=0.75 \mathrm{~s}^{-1}$ that drops less than $5 \mu \mathrm{m}$ are still observable at the bottom of the image (Figure $2 \mathrm{a}^{\prime}$ ). Understanding how this fraction of the population evolves (that is, attempting to elucidate the proper mechanism mediating this capture process) will require experiments with a smaller gap size.

For each subsequent constant shear rate flow section, the duration of the flow for $\sim 400 \mathrm{~s}$ was sufficient to ensure the correct evaluation of the properties of the individual droplets at $\sim 380 \mathrm{~s}$ after start-up of the steady flow. But as a shear rate of $1.5 \mathrm{~s}^{-1}$ was reached, only drops greater than $5 \mu \mathrm{m}$ were observed for $\dot{\gamma}=2.25,3.0,3.75$ and $4.5 \mathrm{~s}^{-1}$ (see Figure $2 \mathrm{~b}^{\prime}-\mathrm{f}^{\prime}$ ). Under the prescribed conditions, all experiments addressed the changes in the size distributions of the particles as a function of (1) the shear rate; (2) the duration of the applied flow; and (3) the possible spatial variation of the distribution of particles parameters.

\section{Results}

Figure 2 shows the images captured during flow and immediately after flow (unprimed and primed labels, respectively-left most columns). The applied shear rates were from 0.75 to $4.5 \mathrm{~s}^{-1}$, top to bottom, respectively, with the corresponding density function of drop sizes—right most column. The objective is to show several of the possible structures in an emulsion induced by flow and observed during these experiments.

Figure 2a shows many small drops, of diameter $<5 \mu \mathrm{m}$ and below the lower limit of the resolution threshold [23], for this optical arrangement; these drops are not included in the distribution for the shear rate of $\dot{\gamma}=0.75 \mathrm{~s}^{-1}$. Indeed, these drops quickly disappear with increasing $(\tau \geq 400)$, as observed in the bottom of the images of Figure $2 b^{\prime \prime}-f^{\prime \prime}$. Possible experiments with a weaker shear rate, $\dot{\gamma} \leq 0.75 \mathrm{~s}^{-1}$, which may be most relevant for the smallest of drops, are not included here and are not considered to be relevant to the observed structural phenomena, which is the main objective of this work. Drops with a diameter larger than $45 \mu \mathrm{m}$ are only a few and were not considered significant to the values of the parameters of the distribution. The relative frequency of drops larger than $45 \mu \mathrm{m}$ is of the order of 0.00172 . 

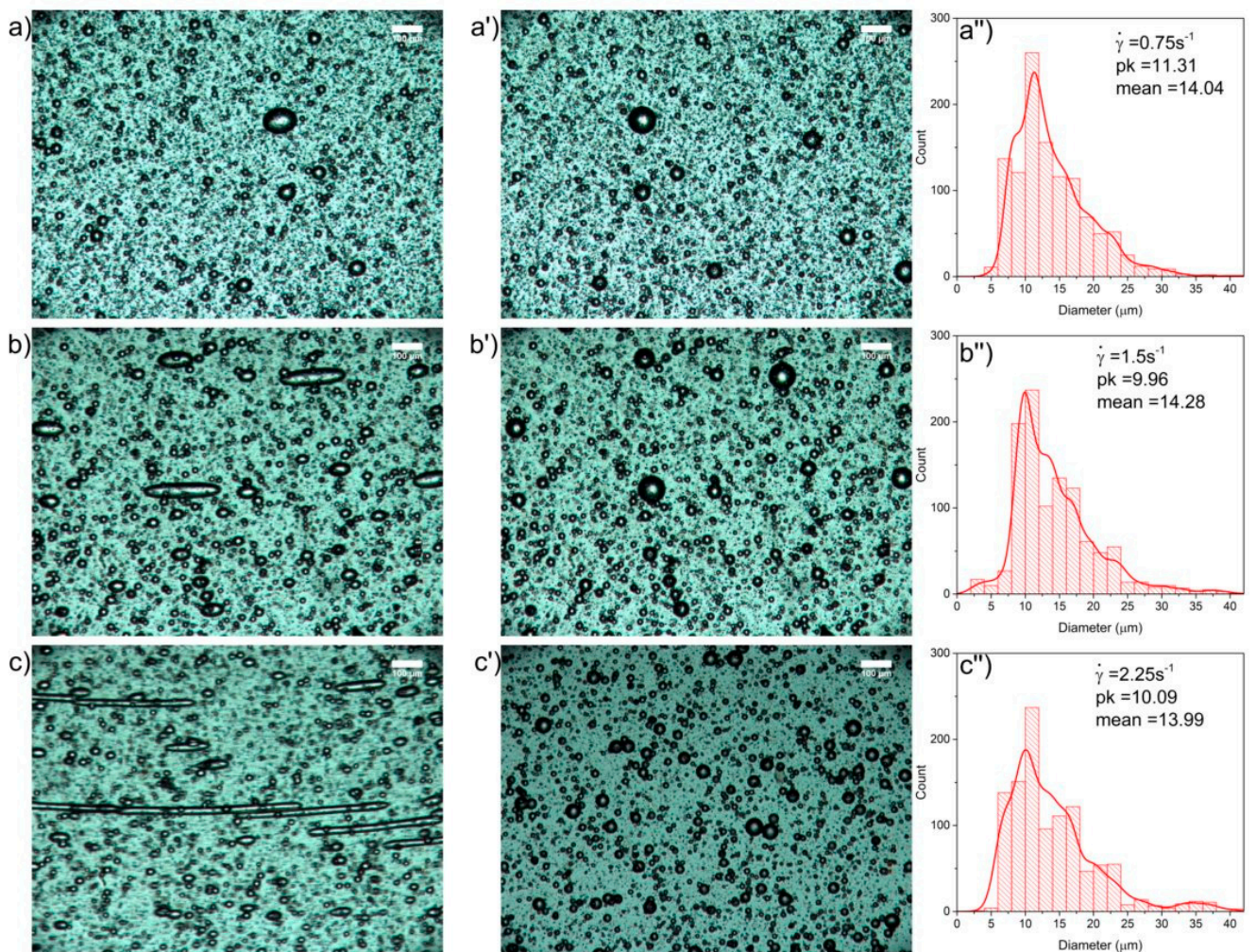

d)
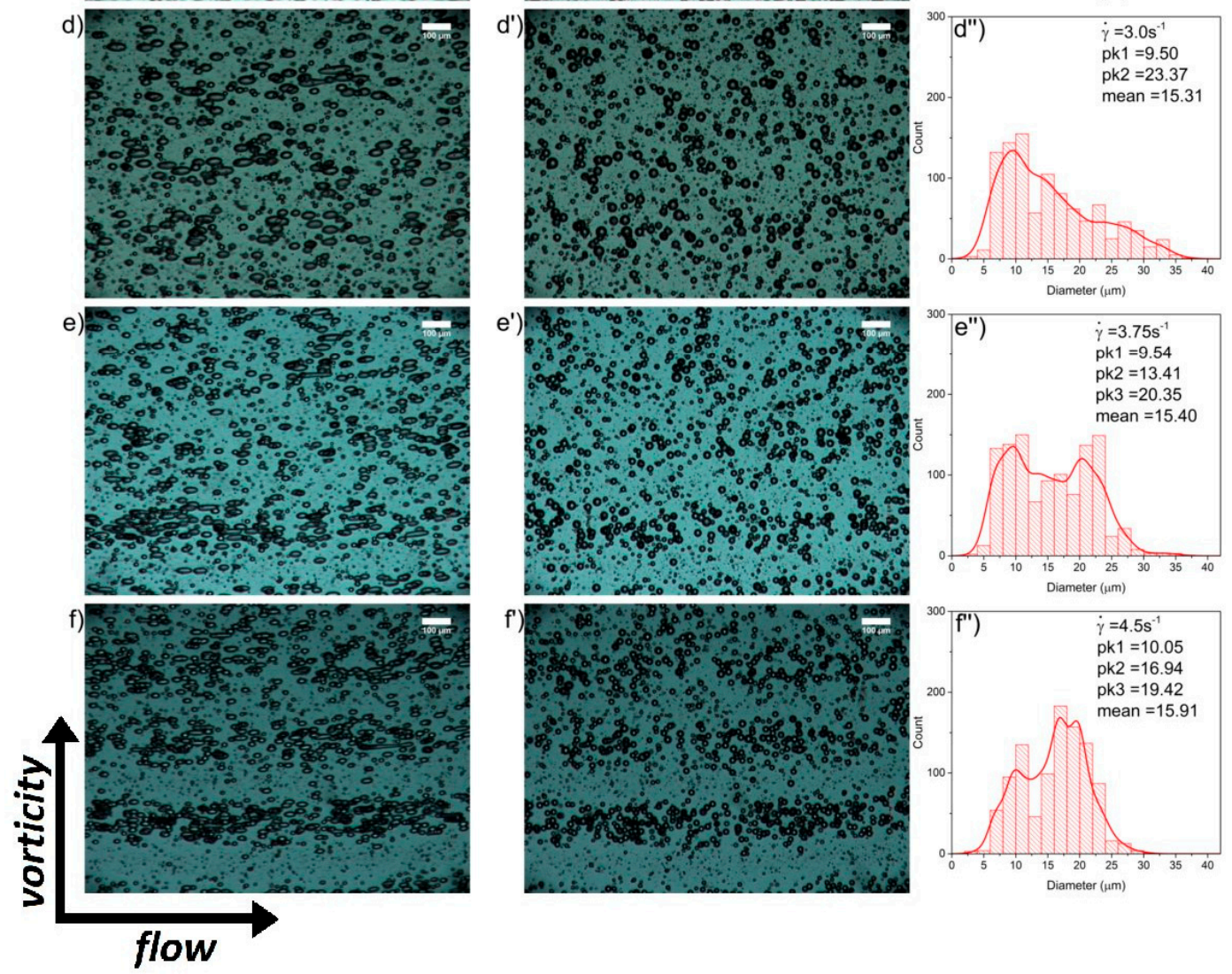

Figure 2. The sequence of images shows the effects due to increase of shear rates (top to bottom) on the evolution of the morphology-during flow, left, unprimed letters (a-f); after cessation of flow $\left(\mathbf{a}^{\prime}-\mathbf{f}^{\prime}\right)$, center, and primed letters-for a (50/50) emulsion. For histograms—right plots, double primes $\left(\mathbf{a}^{\prime \prime}-\mathbf{f}^{\prime \prime}\right)-\mathrm{pk} n$ stands for the first, second, etc., peak of the distribution; mean is the average size of drops. All images are captured with the same magnification and at a temperature of $30^{\circ} \mathrm{C}$. 
One of the first effects of the shearing that was observed that can readily be evaluated occurred for the smallest bin of the population, when the frequency of the smallest of drops (about 5-7.5 $\mu \mathrm{m}$ ) decreased and the frequency of drops with about twice the volume $(8-10 \mu \mathrm{m})$ clearly grew. This condition indicates that for flows with $\dot{\gamma} \leq 2.25 \mathrm{~s}^{-1}$ the critical time for coalescence of the smaller drops already occurred. That is, at $\dot{\gamma}=2.25 \mathrm{~s}^{-1}$, the number of small drops is reduced within a few minutes, with pairs of drops generating larger drops of about twice the volume.

As shown in Figure $2 \mathrm{f}, \mathrm{f}^{\prime}$, at larger shear rates, a poly-disperse emulsion with a multimodal distribution was generated. Here, Figure 2 shows that the average size of drops increased in time as the shear rate increased, accompanied by the formation of a (secondary) peak of larger drops and resulting in a bi-modal distribution. The same coalescence phenomenon appears to dominate the onset of the bi-modal distribution shown in Figure $2 \mathrm{f}^{\prime \prime}$ from smaller size drops. At this flow rate- $\dot{\gamma}=4.5 \mathrm{~s}^{-1}$-the collision of drops with an average diameter close to 12-14 $\mu \mathrm{m}$ gave rise to drops of about $16.5 \mu \mathrm{m}$ (double the volume of the smaller drops). From the histogram in Figure $2 \mathrm{f}^{\prime \prime}$, it is clear that a new population phenomenon was at play: both frequencies of the 10-12 $\mu \mathrm{m}$ and 12-14 $\mu \mathrm{m}$ bins decreased significantly and simultaneously. It appears that pairs of drops of size 10-14 $\mu \mathrm{m}$ may coalesce rapidly, until a more stable drop size is attained, both bins contributing to the appearance of the second and third peaks in the distribution at about $14-20 \mu \mathrm{m}$.

Interestingly, Figure $2 \mathrm{f}$ also shows (a) the onset of pearl collar structures-several drops of similar diameter, evenly spaced and roughly aligned along the flow direction-with ellipsoid-like drop shapes and a waist close to $\sim 15 \mu \mathrm{m}$; and (b) a banded, structured emulsion along the flow direction and perpendicular to the vorticity axis. The surprising feature of the collars and bands were their persistent lengths, several times the characteristic length scale of previous phenomena and several times the thickness of the channel (about $300 \mu \mathrm{m}$ ).

In these experiments, a few large drops were also observed-larger than $40 \mu \mathrm{m}$ in diameter, with an ellipsoid-like shape with an averaged waist size $\sim 35 \mu \mathrm{m}$-at the onset of the flow regime that did not show up initially in the upper tail of the distribution. In Figure 2c, the highly elongated drops-similar to wire-structures - could be attributed to possibly larger drops that had reached a very large deformation, only possibly due to confinement effects by neighboring drops and by the presence of the cell walls, simultaneously.

Table 1 presents the data used for each experiment of the most relevant parameters characterizing these distributions. Thus, approximately 1000 drops were captured for each drop size distribution, and several measures of the diameter were calculated. Of particular relevance are the mean $\left(D_{1,0}\right)$, as well as the Number-area and Number-volume mean diameter. The relevance of these numbers is further expanded upon in the Section 4.

Table 1. Drop size distribution statistics for the histograms shown in Figure 2.

\begin{tabular}{|c|c|c|c|c|c|c|c|c|c|}
\hline \multirow{2}{*}{$\begin{array}{l}\text { Shear } \\
\text { Rate } \\
\left(s^{-1}\right)\end{array}$} & \multirow{2}{*}{$\begin{array}{l}\text { Total } \\
\text { Number } \\
\text { Drops }\end{array}$} & \multirow{2}{*}{$\begin{array}{c}\text { Diameter } \\
\text { Mean } \\
(\mu \mathrm{m})\end{array}$} & \multirow{2}{*}{$\begin{array}{l}\text { Diameter } \\
\text { Median } \\
(\mu \mathrm{m})\end{array}$} & \multirow[t]{2}{*}{$\begin{array}{c}D_{4,3} \\
(\mu \mathrm{m})\end{array}$} & \multirow[t]{2}{*}{$\begin{array}{c}D_{3,2} \\
(\mu \mathrm{m})\end{array}$} & \multirow[t]{2}{*}{$\begin{array}{l}D_{2,0} \\
(\mu \mathrm{m})\end{array}$} & \multirow[t]{2}{*}{$\begin{array}{l}D_{1,0} \\
(\mu \mathrm{m})\end{array}$} & \multicolumn{2}{|c|}{$\begin{array}{l}\text { Distribution } \\
\text { Properties }\end{array}$} \\
\hline & & & & & & & & Skewness & Kurtosis \\
\hline 0.75 & 1148 & 14 & 12.9 & 21.1 & 18.6 & 15 & 13.9 & 1.2 & 1.82 \\
\hline 1.5 & 1078 & 14.3 & 13.2 & 22.8 & 19.7 & 15.6 & 14.4 & 1.32 & 2.46 \\
\hline 2.25 & 1084 & 14 & 13.2 & 24.3 & 20.6 & 15.6 & 14.2 & 1.44 & 2.49 \\
\hline 3 & 1015 & 15.3 & 14.1 & 24.7 & 22.2 & 17.2 & 15.5 & 0.73 & -0.39 \\
\hline 3.75 & 1135 & 15.4 & 15 & 21.9 & 20.3 & 16.8 & 15.6 & 0.26 & -0.86 \\
\hline 4.5 & 1034 & 15.9 & 16.4 & 20 & 19 & 16.9 & 16.2 & -0.12 & -0.61 \\
\hline
\end{tabular}

$D_{4,3}$ is the volume or mass moment mean also known as the De Broucker mean diameter. $D_{3,2}$ is the surface area moment mean or the Sauter mean diameter (SMD). $D_{2,0}$ is the number-area mean diameter. Kurtosis measures the relative weight of the tails with respect to the central portion of the distribution.

In Figure 3, the drop size distributions are shown with their corresponding smoothed kernels for all shear rates studied in this work. The horizontal axis is the bin average diameter and the vertical 
axis is the drop count. It is observed in Figure 3a-c that these size distributions are characterized by a single peak and positive Kurtosis-with a single peak and relatively high weight of the distribution tails (see Table 1). Please note that for a flow cell with a gap of $100 \mu \mathrm{m}$, drops with diameters as large as $100 \mu \mathrm{m}$ should be observed; although, very few are seen during these experiments, which do not significantly alter the Kurtosis of the distribution. Most drop diameters are constrained to less than $40 \mu \mathrm{m}$. In Figure $3 \mathrm{~d}$, the maximum value of $d_{V}=D_{4,3}$ is reached (blue vertical bar). In Figure 3e,f, a rapid decrease in the value of $D_{4,3}$ (Figure 4) is shown. This effect is mainly due to a rather broad peak, which here becomes evident by the presence of a second peak that emerges from right to left, and a Kurtosis measure that changes sign. For the drop size distribution generated when $\dot{\gamma}=4.5 \mathrm{~s}^{-1}$, the skewness parameter becomes negative indicating a larger (secondary) peak at higher diameters.

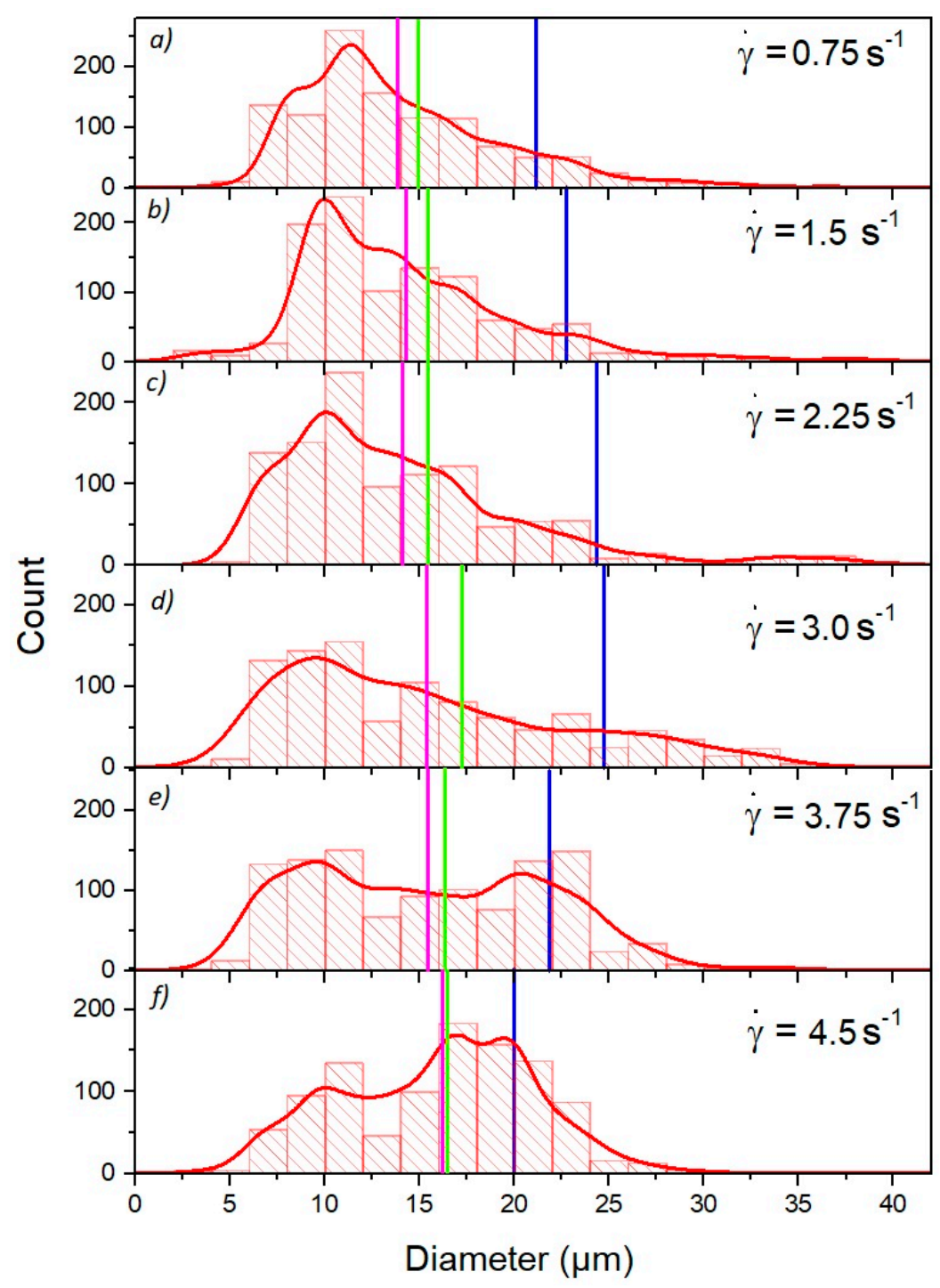

Figure 3. Droplet size distribution and the smoothing kernels for all shear rates studied. For the first three (lower) shear rates $(\mathbf{a}-\mathbf{c})$, the size distributions are characterized by a single peak and positive Kurtosis [34-37]; with rather broad distributions. The magenta bar corresponds to the mean diameter for the distribution, the green bar to the $D_{2,0}$ value, and the blue bar corresponds to the value of $D_{4.3}$. In histogram (d), the maximum value of $D_{4.3}$ is reached (see Table 1 ). Histograms (e,f) show a rapid decrease in the value of $D_{4.3}$, characteristic of distributions with a high top and a smaller contribution from outliers. 
Figure 4 presents the evolution of the mean drop size using the suggested averages (values shown in Table 1). Figure 4a presents the diameter evolution with respect to the dimensionless time (or as a function of the applied shear strain by the flow); Figure $4 \mathrm{~b}$ corresponds to the same data vs. the applied shear rate. It is clear that $D_{1,0}$ and $D_{2,0}$ remain essentially constant at 14 and $15.5 \mu \mathrm{m}$, respectively, for times less than $\tau \leq 800$, abruptly increasing to means of 15.5 and $17 \mu \mathrm{m}$, respectively, under a faster flow and a longer duration of the flow.

Figure $4 \mathrm{c}, \mathrm{d}$ correspond to the normalized average size $\left(D_{4,3} / D_{1,0}\right.$ and $\left.D_{2,0} / D_{1,0}\right)$ versus the dimensionless time and the accumulated dimensionless time (total imposed strain). Therefore, the base line for these two plots corresponds to $D_{1,0}$, a two-step function with rather constant lower and upper values (i.e., $14 \mu \mathrm{m}$ and $15.5 \mu \mathrm{m}$ (see Figure 4a)). The droplets generated during the preparation of the emulsion grew constantly within the initial 675 units of dimensionless time and then evolved rapidly to an average mean diameter of $15.5 \mu \mathrm{m}$. In most experiments, close to 300-10,000 units of dimensionless time are necessary to reach a reproducible size distribution of the emulsions. The longer times are especially the case for global anisotropic structures, which may require a total dimensionless time longer than 8000, as shown in Figure 4d.
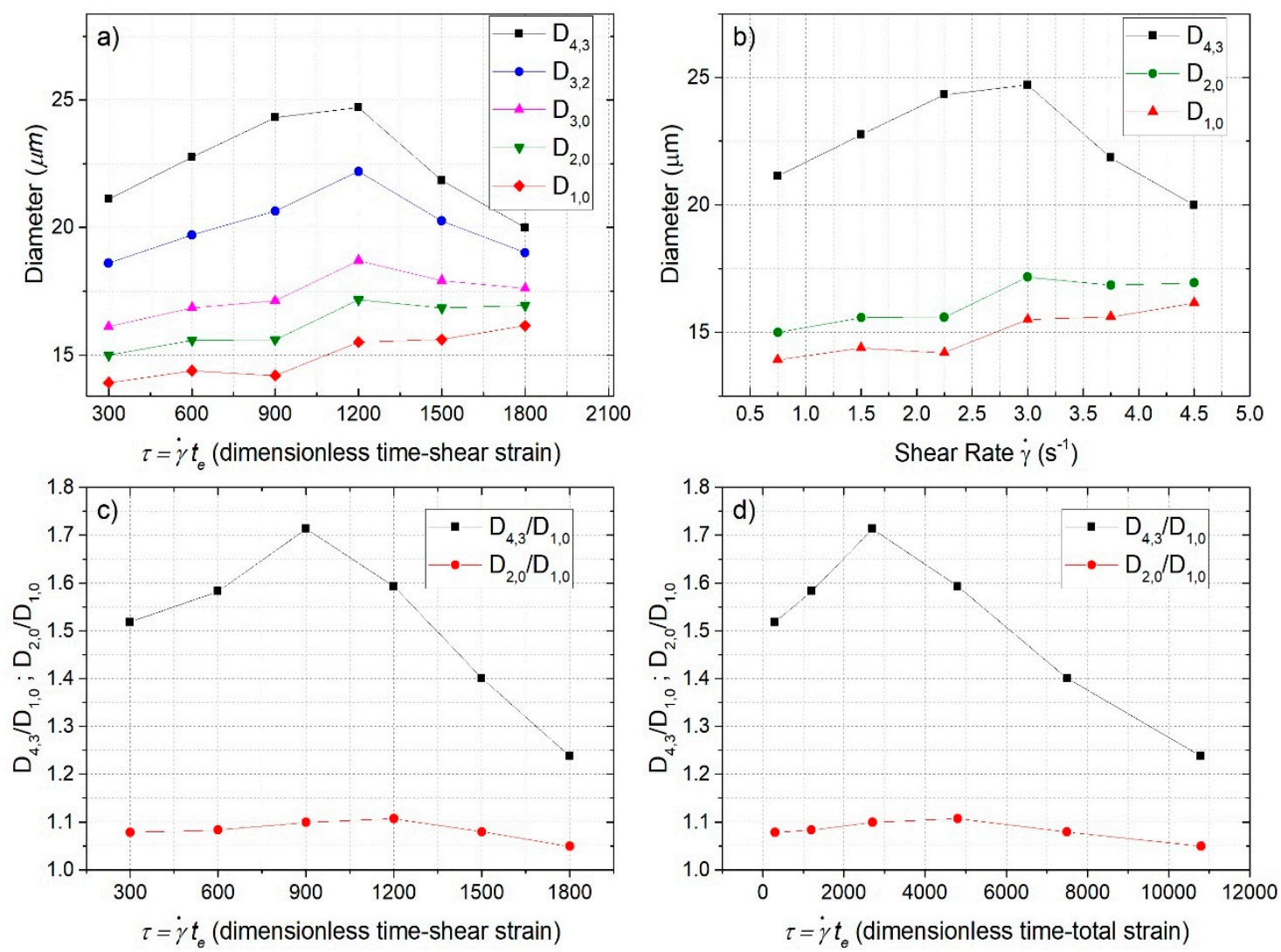

Figure 4. (a) Mean diameters of the distributions $-D_{1,0}$ up to $D_{4,3}-$ vs. the dimensionless time $\tau_{i}$ incurred during each flow regime; (b) $D_{1,0}, D_{2,0}$, and $D_{4,3}$ vs. the applied shear rate during each regime; $(\mathbf{c}, \mathbf{d})$ are, respectively, the dimensionless normalized mean drop size vs dimensionless time in each cycle and for the total duration of the flow $\sum \dot{\gamma}_{i} t_{i}$.

\section{Discussion}

The distributions of drops appear to change mainly due to two processes, coalescence and rupture, with little evidence of Oswald ripening. The lack of evidence for the latter may be due to the fact that drops larger than $35-40 \mu \mathrm{m}$ were not observed. In contrast, the presence of the coalescence phenomenon can be inferred at several instances in the evolution of the distributions, which may 
be due to different hydrodynamic processes albeit operating simultaneously. As pointed out in the description of the coalescence kinetics, the most likely case corresponds to two of the smaller drops (about equal size) generating a drop with about twice their volume. This was clearly the case for the smaller drops in Figure 3a-d. But coalescence may also be the dominant mechanism for the reduction of probability observed in the first peak and in the dawning of the second peak. During collision of these larger drops, those of $\sim 10 \mu \mathrm{m}$ disappear, with the $15 \mu \mathrm{m}$ bin growing and during a subsequent coalescence, becoming drops of about $20 \mu \mathrm{m}$. Coalescence seems to be the dominant mechanism for critical dimensionless time less than $\tau \leq 700$, with an associated critical shear rate of about $\dot{\gamma} \leq 2.25 \mathrm{~s}^{-1}$. Coalescence occurs simultaneously with rupture up to $\tau \approx 8000 \dot{\gamma} \leq 4.5 \mathrm{~s}^{-1}$.

A second possible mechanism for the onset of the secondary peak is break up, particularly for the heavier tail, with the disappearance of the much larger drops (less than $40 \mu \mathrm{m}$ ) producing smaller drops of about half their volume (i.e., $\sim 27 \mu \mathrm{m}$ (as shown when $\dot{\gamma}=3.0-3.75 \mathrm{~s}^{-1}$ (Figure 3d,f))). These drops, in turn, break up again, producing an increase in frequency for drops sizes of about $22 \mu \mathrm{m}$. The rupture mechanism appears to depend on dimensionless time and on a critical shear rate of the order of $\dot{\gamma}=3.0-3.75 \mathrm{~s}^{-1}$, not being observed for smaller drops. The simultaneous contribution of these two mechanisms makes it possible to explain the appearance of the second peak of the bimodal distribution.

Figure 4 presents the evolution of the mean drop size (with five different measures) versus its shear rate history. Initially, droplets generated during the preparation of the emulsion grew constantly, from sizes less than the threshold of the image processing, but attained values that can be readily evaluated with the statistical measures proposed for $\tau \geq 300$, as shown in Figure 4. The mean value $D_{1,0}$ remained at a constant value for shear rates less than $\dot{\gamma} \leq 2.25 \mathrm{~s}^{-1}$-the same behavior is observed for $D_{2,0}$-while $D_{3,2} D_{4,3}$ increased linearly with dimensionless time up to $900 \tau$ units, followed by a monotonic decrease of the mean size for the total accumulated dimensionless time $\tau \geq 3000$.

For dimensionless time between 675-900 and under an increment of $\dot{\gamma}>2.25 \mathrm{~s}^{-1}$, the mean value $D_{1,0}\left(\right.$ and $\left.D_{2,0}\right)$ appeared to remain at a new constant value of $D_{1,0}=15.5 \mu \mathrm{m}$ (and $\left.D_{2,0}=17.5 \mu \mathrm{m}\right)$ approximately. This jump in mean diameter correlated with the time and shear rate in these experiments. An interesting observation is that the ratio of these two means was essentially constant for the complete range of values of the shear rate (see Figure $4 \mathrm{c}$ ) regardless of the dimensionless time or shear rate. In contrast, measures $D_{3,2}$ and $D_{4,3}$ did change significantly ( $-25 \%$ ) after $\tau \geq 675$ or under an increase of $\dot{\gamma}>3.0 \mathrm{~s}^{-1}$. This abrupt collapse of $D_{3,2}$ and $D_{4,3}$ values correlated with the behavior of other statistical measures, indicating a dominant breakup mechanism of the larger drops. The rupture mechanism was most clearly associated with the appearance of pairs of satellite drops (of about half volume) that did not appear to influence the low end of the distribution. Hence, under these flows, the drops attained deformations associated with dumbbell shapes before breaking up, with a few of them reaching a significantly elongated waist capable of generating smaller satellite drops.

In most cases, 100-10,000 units of strain are necessary to reach a size distribution of the emulsion with low tail populations. Figure $4 \mathrm{~d}$ shows a total time longer than $\tau \geq 8000$, a time scale that is representative of the anisotropic global structures observed in this emulsion. By increasing the shear rate, the arithmetic average value increased at a rather constant rate. However, the position of the peaks was not affected in a forceful way and appeared to be independent of time, contrary to the behavior of the width of each of the main peaks, which became broader over time while keeping the area under the curve approximately equal. This phenomenon may have been the result of the disappearance of the smallest droplets, as shown in Figure 3f. For the large drops, the formation of a secondary peak from right to left was observed (see Figure $3 d-f$ ), resulting in the decrease of the height of the first peak and the increase of its width and vice versa for the second peak.

Figure $4 \mathrm{~d}$ shows a total time longer than $\tau \geq 8000$. In other to evaluate in detail the global structure shown in Figure 2e,f, associated with $\tau \sim 8000$ global times, future experiments may require times significantly longer than the length of time considered here, as well as a new statistical analysis for evaluation of the anisotropic character of the distribution of particles in the sample. 


\section{Conclusions}

This emulsion showed good stability when left to rest. However, under flow conditions, the size distribution changed significantly in terms of the mean values, as well as other characteristics, such as mono- to bimodal distributions or measures of skewness and kurtosis. Besides coalescence and rupture as possible explanations of the observed behavior at lower shear rates, other possible structures are readily observable, such as necklaces and banded structures. Evidence of necklaces appear at a transition $\dot{\gamma} \sim 3.0 \mathrm{~s}^{-1}$ in a single band to transient chains of drops in the flow direction and in several layers at a shear rate of $\dot{\gamma} \sim 3.75 \mathrm{~s}^{-1}$.

These experiments also elucidate two possible mechanisms for the evolution of the size distribution of drops in highly concentrated emulsions at low shear rates. The first is coalescence, dominant for smaller drops at low shear rates and short times. The arithmetic mean of 14.0-14.3 $\mu \mathrm{m}$ under a shear rate of about $0.75-1.5 \mathrm{~s}^{-1}$ can be explained by coalescence of the smallest drops, while indicating a poor stability to flow induced perturbations. The behavior of the small drops indicates a high frequency of close neighbor encounters, promoting coalescence during this stage. Due to the fact that the efficiency of coalescence decreases with the increasing difference in the size of the colliding drops [2,24], coalescence tends to be less likely at longer times. These results are in agreement with the qualitative works of Grizzuti and Bifulco [25] and Rusu and Peuvrel-Disdier [23].

The weak increase of the $D_{1,0}$ value of the distribution as the shear rate grows may indicate that it is independent of time, which is in contrast to the behavior of the width of each of the main peaks, which increase as time passes while keeping the area under the curve approximately constant. Observing the evolution of the peaks and width of peaks in Figure $3 d-f$, it becomes evident that the largest drops rupture, giving rise to smaller ones. These dynamics may induce the appearance of the second peak (of larger drops) in Figure 3f. Here, Figure $4 \mathrm{c}$, $\mathrm{d}$ show that the $D_{4,3}$ measure decreases significantly relative to the mean value when the tail of the distribution for large drops is decreasing and rupture is a prevalent mechanism. This hypothesis may be correct; although, we have not addressed this point in detail, we may do it in future work. The tails of the distribution are less dominant as shown by the skewness and kurtosis values.

Author Contributions: J.M.L. and E.G. conceived of and designed the experiments; J.M.L. performed the experiments; J.M.L. and E.G. analyzed the data; E.G. contributed reagents/materials/analysis tools; and J.M.L. and E.G. wrote the paper.

Funding: This research was funded by DGAPA-UNAM, Mexico; grant number PAPIIT-IN114618.

Acknowledgments: We thank the reviewers for their insightful comments and suggestions. J.M.L. thanks for financial support throughout his Ph.D. studies in Materials Science and Engineering, UNAM, to Consejo Nacional de Ciencia y Tecnología (CONACYT, Mexico).

Conflicts of Interest: The authors declare no conflict of interest. Sponsors had no role in the design of the study; in the data collection, analyses, or interpretation of data; in the writing of the manuscript; or in the decision to publish the results.

\section{References}

1. Berg, J.C. Fluid interfaces and capillarity. In An Introduction to Interfaces \& Colloids: The Bridge to Nanoscience; World Scientific: Singapore, 2010; pp. 30-80, ISBN 978-9-81-429307-5.

2. Park, J.Y.; Blair, L.M. The effect of coalescence on drop size distribution in agitated liquid-liquid dispersion. Chem. Eng. Sci. 1975, 30, 1057-1064. [CrossRef]

3. Sundararaj, U.; Macosko, C.W. Drop breakup and coalescence in polymer blends: The effects of concentration and compatibilization. Macromolecules 1995, 28, 2647-2657. [CrossRef]

4. Leal, L.G. Flow induced coalescence of drops in a viscous fluid. Phys. Fluids 2004, 16, 1833-1851. [CrossRef]

5. De Bruijn, R.A. Tipstreaming of drops in simple shear flows. Chem. Eng. Sci. 1993, 48, 277-284. [CrossRef]

6. Rumscheidt, F.D.; Mason, S.G. Particle motions in sheared suspensions XII. Deformation and burst of fluid drops in shear and hyperbolic flow. J. Colloid Sci. 1961, 16, 238-261. [CrossRef] 
7. Rumscheidt, F.D.; Mason, S.G. Particle motions in sheared suspensions XI. Internal circulation in fluid droplets (experimental). J. Colloid Sci. 1961, 16, 210-237. [CrossRef]

8. Delaby, I.; Ernst, B.; Germain, Y.; Muller, R. Droplet deformation in polymer blends during uniaxial elongational flow: Influence of viscosity ratio for large capillary numbers. J. Rheol. 1994, 38, 1705-1720. [CrossRef]

9. Caserta, S.; Sabetta, L.; Simeone, M.; Guido, S. Shear-induced coalescence in aqueous biopolymer mixtures. Chem. Eng. Sci. 2005, 60, 1019-1027. [CrossRef]

10. Frith, W.J.; Lips, A. The rheology of concentrated suspensions of deformable particles. Adv. Colloid Interface Sci. 1995, 61, 161-189. [CrossRef]

11. Vinckier, I.; Moldenaers, P.; Terracciano, A.M.; Grizzuti, N. Droplet size evolution during coalescence in semiconcentrated model blends. AIChE J. 1998, 44, 951-958. [CrossRef]

12. Lyu, S.P.; Bates, F.S.; Macosko, C.W. Coalescence in polymer blends during shearing. AIChE J. 2000, 46, 229-238. [CrossRef]

13. Taylor, G.I. The viscosity of a fluid containing small drops of another fluid. Proc. R. Soc. Lond. A. 1932, 138, 41-48. [CrossRef]

14. Utracki, L.A.; Shi, Z.H. Development of polymer blend morphology during compounding in a twin-screw extruder. Part I, Droplet dispersion and coalescence-A review. Polym. Eng. Sci. 1992, 32, 1824-1833. [CrossRef]

15. Torza, S.; Cox, R.G.; Mason, S.G. Particle motions in sheared suspensions XXVII. Transient and steady deformation and burst of liquid drops. J. Colloid Interface Sci. 1972, 38, 395-411. [CrossRef]

16. Grace, H.P. Dispersion phenomena in high viscosity immiscible fluid systems and application of static mixers as dispersion devices in such systems. Chem. Eng. Commun. 1982, 14, 225-277. [CrossRef]

17. De Bruijn, R.A. Deformation and Breakup of Drops in Simple Shear Flows. Ph.D. Thesis, Eindhoven University of Technology, Eindhoven, The Netherlands, 1989.

18. Chesters, A. Modelling of coalescence processes in fluid-liquid dispersions: A review of current understanding. Chem. Eng. Res. Des. 1991, 69, 259-270.

19. Abid, S.; Chesters, A.K. The drainage and rupture of partially-mobile films between colliding drops at constant approach velocity. Int. J. Multiph. Flow 1994, 20, 613-629. [CrossRef]

20. Coulaloglou, C.A.; Tavlarides, L.L. Description of interaction processes in agitated liquid-liquid dispersions. Chem. Eng. Sci. 1977, 32, 1289-1297. [CrossRef]

21. Ramic, A.J.; Stehlin, J.C.; Hudson, S.D.; Jamieson, A.M.; Manas-Zloczower, I. Influence of block copolymer on droplet breakup and coalescence in model immiscible polymer blends. Macromolecules 2000, 33, 371-374. [CrossRef]

22. Liao, Y.; Lucas, D. A Literature review on mechanisms and models for the coalescence process of fluid particles. Chem. Eng. Sci. 2010, 65, 2851-2864. [CrossRef]

23. Rusu, D.; Peuvrel-Disdier, E. In Situ characterization by small angle light scattering of the shear-induced coalescence mechanisms in immiscible polymer blends. J. Rheol. 1999, 43, 1391-1409. [CrossRef]

24. Lyu, S.; Bates, F.S.; Macosko, C.W. Modeling of coalescence in polymer blends. AIChE J. 2002, 48, 7-14. [CrossRef]

25. Grizzuti, N.; Bifulco, O. Effects of coalescence and breakup on the steady-state morphology of an immiscible polymer blend in shear flow. Rheol. Acta 1997, 36, 406-415. [CrossRef]

26. Minale, M.; Mewis, J.; Moldenaers, P. Study of the morphological hysteresis in immiscible polymer blends. AIChE J. 1998, 44, 943-950. [CrossRef]

27. Minale, M.; Moldenaers, P.; Mewis, J. Effect of shear history on the morphology of immiscible polymer blends. Macromolecules 1997, 30, 5470-5475. [CrossRef]

28. Bentley, B.J.; Leal, L.G. An experimental investigation of drop deformation and breakup in steady, two-dimensional linear flows. J. Fluid Mech. 1986, 167, 241-283. [CrossRef]

29. Jansen, K.M.; Agterof, W.G.; Mellema, J. Droplet breakup in concentrated emulsions. J. Rheol. 2001, 45, 227-236. [CrossRef]

30. Guido, S.; Villone, M. Measurement of interfacial tension by drop retraction analysis. J. Colloid Interface Sci. 1999, 209, 247-250. [CrossRef] [PubMed]

31. Silverman, B.W. The kernel method for univariate data. In Density Estimation for Statistics and Data Analysis, 1st ed.; Routledge: New York, NY, USA, 1986; pp. 34-74, ISBN 0412246201. 
32. Scott, D.W. Kernel Density Estimators. In Multivariate Density Estimation: Theory, Practice, and Visualization, 2nd ed.; John Wiley \& Sons: Hoboken, NJ, USA, 2015; pp. 137-216, ISBN 1118575482.

33. Caserta, S.; Simeone, M.; Guido, S. 3D optical sectioning and image analysis of particles in biphasic systems. Microsc. Anal. 2005, 19, 9-11.

34. Wang, H. Path factors of bipartite graphs. J. Graph Theory 1994, 18, 161-167. [CrossRef]

35. Kurtosis in Probability Distributions. Available online: https://en.wikipedia.org/wiki/Kurtosis (accessed on 22 April 2018).

36. Kim, T.H.; White, H. On more robust estimation of skewness and kurtosis. Financ. Res. Lett. 2004, 1, 56-73. [CrossRef]

37. Moments. Available online: https://www.originlab.com/doc/X-Function/ref/moments (accessed on 22 April 2018).

2018 by the authors. Licensee MDPI, Basel, Switzerland. This article is an open access article distributed under the terms and conditions of the Creative Commons Attribution (CC BY) license (http://creativecommons.org/licenses/by/4.0/). 